\title{
Decrease in matrix metalloproteinase-3 activity in systemic sclerosis fibroblasts causes $\alpha 2$-antiplasmin and extracellular matrix deposition, and contributes to fibrosis development
}

\author{
HIROFUMI NIWA $^{1}$, YOSUKE KANNO ${ }^{1,2}$, EN SHU $^{1}$ and MARIKO SEISHIMA ${ }^{1}$ \\ ${ }^{1}$ Department of Dermatology, Gifu University Graduate School of Medicine, Gifu 501-1194; \\ ${ }^{2}$ Department of Clinical Pathological Biochemistry, Faculty of Pharmaceutical Science, \\ Doshisha Women's College of Liberal Arts, Kyoto 610-0395, Japan
}

Received February 7, 2020; Accepted July 1, 2020

DOI: $10.3892 / \mathrm{mmr} .2020 .11358$

\begin{abstract}
Systemic sclerosis ( $\mathrm{SSc}$ ) is a connective tissue disease of autoimmune origin characterized by fibrosis of the skin and visceral organs, and peripheral circulatory disturbance. $\alpha 2$-antiplasmin ( $\alpha 2 \mathrm{AP})$ is the major circulating inhibitor of plasmin and is a key regulator of fibrinolysis. It has been demonstrated that the expression of $\alpha 2 \mathrm{AP}$ is elevated in dermal fibroblasts obtained from patients with SSc patients. It has also been determined that $\alpha 2 \mathrm{AP}$ is associated with the development and progression of fibrosis in SSc. The present study assessed the relationship between $\alpha 2 \mathrm{AP}$ and matrix metalloproteinase- 3 (MMP-3), an extracellular matrix (ECM)-degrading enzyme. Serum levels of $\alpha 2 \mathrm{AP}$ and MMP-3 were measured in healthy controls and patients with SSc using ELISA. No significant differences were determined between these two groups. $\alpha 2 \mathrm{AP}, \mathrm{MMP}-3$ and tissue inhibitor of metalloproteinase-1 (TIMP-1) expression was subsequently evaluated in normal and SSc fibroblasts via western blotting. The results revealed that $\alpha 2 \mathrm{AP}$ expression increased in SSc dermal fibroblasts, while the ratio of MMP-3/TIMP-1 decreased. Additionally, incubation of recombinant $\alpha 2 \mathrm{AP}$ with MMP-3 caused $\alpha 2 \mathrm{AP}$ degradation. The mixture of recombinant $\alpha 2 \mathrm{AP}$ with MMP-3 was subsequently added to normal fibroblasts prior to western blotting. The results revealed decreased $\alpha$-smooth muscle actin $(\alpha$-SMA; a marker of the myofibroblast phenotype) and type I collagen expression. The stimulation of SSc fibroblasts with
\end{abstract}

Correspondence to: Dr Hirofumi Niwa, Department of Dermatology, Gifu University Graduate School of Medicine, 1-1 Yanagido, Gifu 501-1194, Japan

E-mail: h_niwa@gifu-u.ac.jp

Abbreviations: SSc, systemic sclerosis; $\alpha 2 \mathrm{AP}, \alpha 2$-antiplasmin; ECM, extracellular matrix; TIMP-1, tissue inhibitor of metalloproteinase-1

Key words: alpha2-antiplasmin, Systemic sclerosis, Fibrosis, matrix metallopeptidase-3, tissue inhibitor of metalloproteinase-1
MMP-3 decreased protein levels of $\alpha 2 \mathrm{AP}, \alpha$-SMA and type I collagen, thus reversing the pro-fibrotic phenotype of SSc fibroblasts. SSc fibroblast transfection with microRNA-29a resulted in a decreased TIMP-1 expression, but also decreased the protein expression of $\alpha 2 \mathrm{AP}$. The results indicated that MMP-3 attenuated fibrosis progression by degrading $\alpha 2 \mathrm{AP}$ and ECM, and might therefore contribute to a novel therapeutic approach for SSc treatment.

\section{Introduction}

Systemic sclerosis (SSc) is a chronic connective tissue disease that causes widespread microvascular damage and excessive collagen deposition in the skin and internal organs (1). However, the etiology, pathogenesis, and progression of this disease are not fully understood.

Alpha2-antiplasmin ( $\alpha 2 \mathrm{AP})$, is a $65-70 \mathrm{kDa}$ protein that inactivates plasmin and thereby inhibits fibrinolysis $(2,3)$. a2AP exists in various tissues, such as the liver, kidney, intestine, spleen, lung, muscle, ovary, testis, cerebral cortex, hippocampus, cerebellum, bone, skin, and placenta of murine tissue (4). Apart from the inhibition of plasmin, $\alpha 2 \mathrm{AP}$ regulates various cell functions, including proliferation, differentiation, and cytokine production, and also associates with angiogenesis, tissue repair, vascular remodeling, and fibrosis progression (5-13). In patients with rheumatic diseases, including SSc, plasma levels of the plasmin- $\alpha 2 \mathrm{AP}$ complex are increased (14,15). $\alpha 2 \mathrm{AP}$ affects myofibroblast differentiation, extracellular matrix (ECM) production, vascular dysfunction, and progression of SSc. In addition, we have shown that $\alpha 2 \mathrm{AP}$ levels are elevated in an SSc mouse model and dermal fibroblasts $(16,17)$.

Matrix metalloproteinase-3 (MMP-3) plays a pivotal role in ECM turnover as it can degrade ECM components, including proteoglycans, collagen III, IV, V, and IX, laminin, fibronectin, gelatin, and elastin $(18,19)$. MMP-3 is expressed by fibroblasts, chondrocytes, osteoblasts, endothelial cells, smooth muscle cells and macrophages (20). Jinnin et al (21) observed similar MMP-3 serum levels in SSc patients and healthy controls; however, serum levels of anti-MMP-3 autoantibody and tissue inhibitors of metalloproteinase-1 (TIMP-1) were higher in SSc 
patients, suggesting that MMP-3 activity may be decreased in SSc $(20,22)$. Since it has been reported that cleavage by MMP-3 inactivates $\alpha 2 \mathrm{AP}$ (23), the suppression of MMP-3 activity in SSc may promote the activation of $\alpha 2 \mathrm{AP}$.

In the present study, we investigated the relationship between $\alpha 2 \mathrm{AP}$ and MMP-3 to gain insights into the pathogenesis of SSc.

\section{Materials and methods}

The experiments with human samples in this study were approved by the Gifu University Graduate School of Medicine Ethics Committee (Approved ID:29-152). We received written informed consent from the patients and volunteers involved.

Cell culture. Human normal and SSc dermal fibroblasts were obtained from seven patients with SSc and four healthy controls as previously described (6). Fibroblasts were seeded onto 60-mm diameter dishes and cultured in $2 \mathrm{ml}$ Dulbecco's modified Eagle's medium (DMEM) containing 10\% fetal calf serum (FCS) at $37^{\circ} \mathrm{C}$ in a humidified atmosphere with $5 \%$ $\mathrm{CO}_{2} / 95 \%$ air. After 6 days, the medium was replaced with serum-free DMEM. Human normal dermal fibroblasts were stimulated by $\alpha 2 \mathrm{AP}, \mathrm{MMP}-3$ or mixture of $\alpha 2 \mathrm{AP}$ and MMP-3 for $24 \mathrm{~h}$. In other studies, human SSc dermal fibroblasts were stimulated by MMP-3 for $24 \mathrm{~h}$.

Western blot analysis. Cells were washed twice with cold PBS, harvested, and then sonicated in lysis buffer containing $10 \mathrm{mM}$ Tris- $\mathrm{HCl}$ buffer (pH 7.5), $1 \%$ SDS, $1 \%$ Triton X-100, and a protease inhibitor cocktail (Roche Diagnostics $\mathrm{GmbH}$ ). The skin samples from the subjects were homogenized and sonicated in the lysis buffer. The protein concentration in each lysate was measured using a BCA protein assay kit (Pierce; Thermo Fisher Scientific). Proteins in the supernatant were separated by electrophoresis on $10 \%$ SDS-polyacrylamide gels and transferred to a PVDF membrane. We detected each protein by incubation with the relevant primary antibodies followed by horseradish peroxidase-conjugated antibodies to IgG.

Enzyme-linked immunosorbent assay (ELISA). Blood samples were obtained from $10 \mathrm{SSc}$ patients and 10 healthy volunteers, and were subsequently centrifuged for $10 \mathrm{~min}$ at $1,600 \mathrm{xg}$. The supernatant was then collected and used for the assay. The serum levels of $\alpha 2 \mathrm{AP}$ and MMP-3 were determined using ELISA kits, Human Serpin F2/ $\alpha$ 2-Antiplasmin (R\&D Systems, MN, USA) and Human Total MMP-3 Immunoassay (R\&D Systems), respectively. The absorbance was measured at $450 \mathrm{~nm}$ using an iMark Microplate Reader (Bio-Rad Laboratories, Inc.).

miRNA study. SSc dermal fibroblasts were transfected with miR-29a (sequence: ACUGAUUUCUUUUGGUGUUCAG, Bioneer) or negative control miRNA using Lipofectamine 2000 (Invitrogen; Thermo Fisher Scientific) according to the manufacturer's instructions. Cells were harvested $48 \mathrm{~h}$ after transfection for further analysis.

Statistical analysis. All data were expressed as mean \pm SEM and analyzed using Statmate III version 3.06 (ATMS Co., Ltd.).
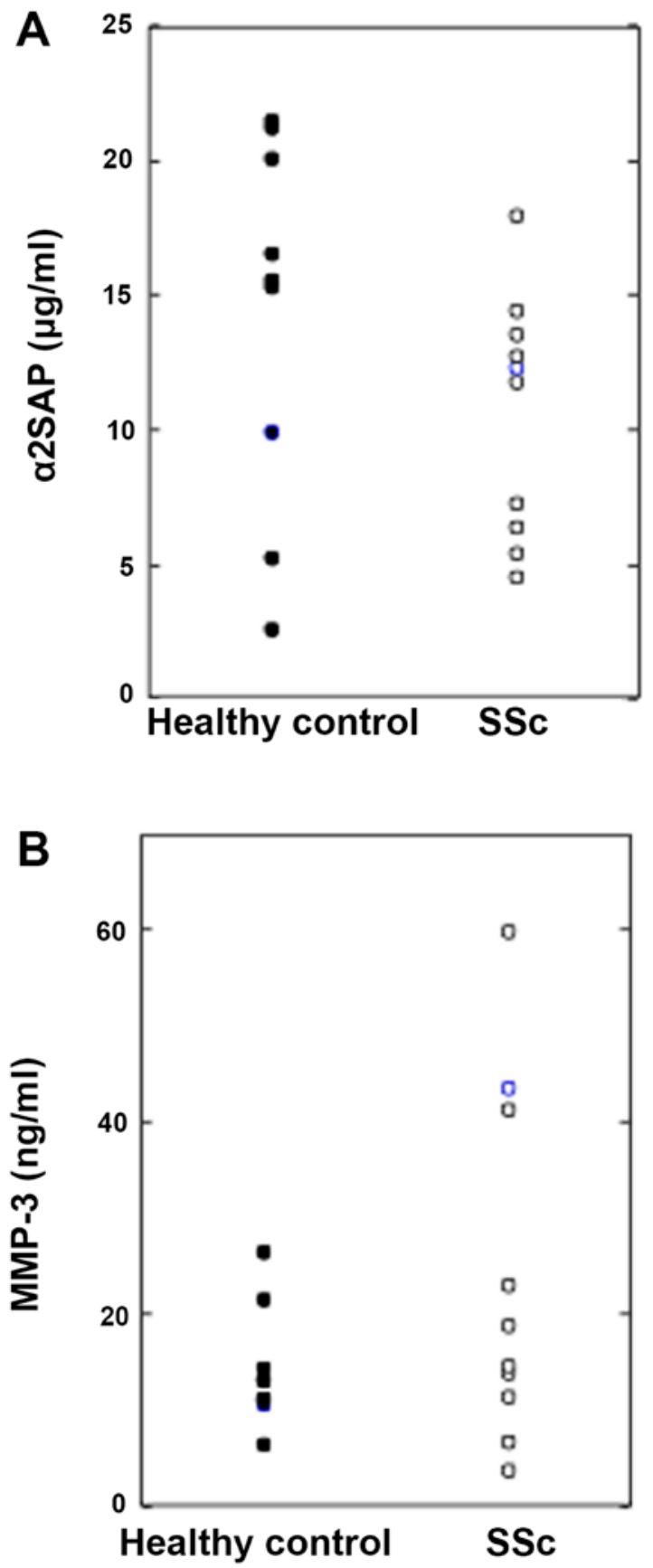

Figure 1. Serum levels of $\alpha 2 \mathrm{AP}$ and MMP-3 in patients with SSc and healthy controls. Blood samples were obtained from 10 patients with SSc and 10 healthy volunteers, and subsequently centrifuged for $10 \mathrm{~min}$ at $1,600 \mathrm{x} \mathrm{g}$ and $4^{\circ} \mathrm{C}$. Supernatants were then collected and used for assessment. The serum levels of (A) $\alpha 2 \mathrm{AP}$ and (B) MMP-3 were measured using ELISA. No significant differences were identified between patients and healthy controls. Data are presented as the mean \pm SEM. $\alpha 2 \mathrm{AP}, \alpha 2$-antiplasmin; MMP-3, matrix metallopeptidase-3; SSc, systemic sclerosis.

The statistical analysis was conducted with unpaired t-test for two-group comparisons, with one-way ANOVA Tukey's for multiple comparison. $\mathrm{P}<0.05$ was considered to indicate a statistically significant difference.

\section{Results}

Serum levels of a2AP and MMP-3 are similar in healthy controls and SSc patients. We examined the levels of $\alpha 2 \mathrm{AP}$ 

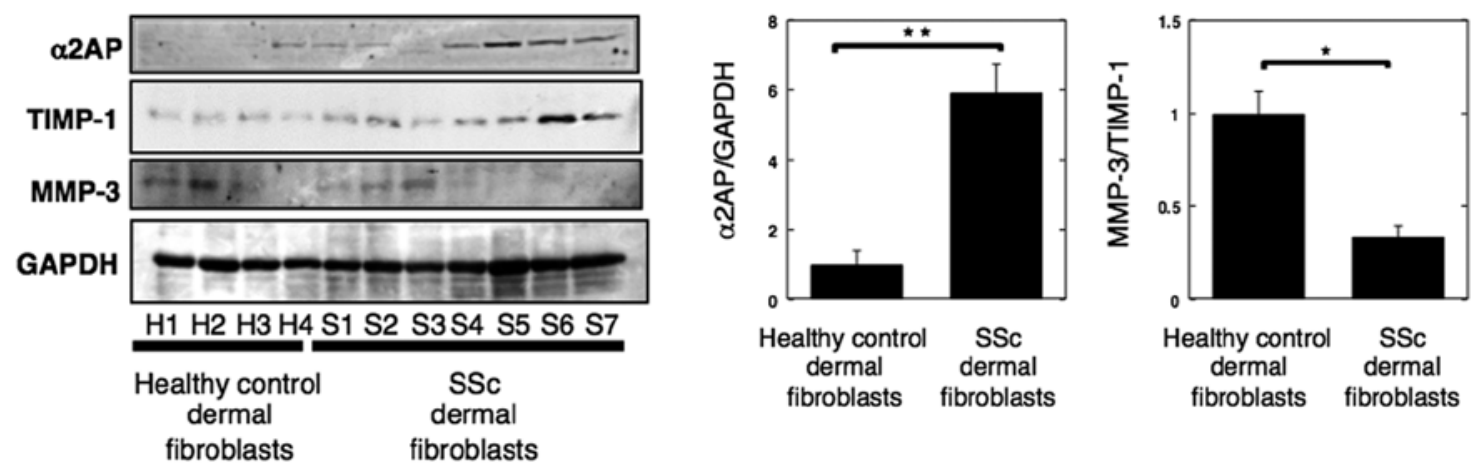

Figure 2. $\alpha 2 \mathrm{AP}$ expression and MMP-3/TIMP-1 ratio in human normal and SSc dermal fibroblasts. Protein levels of $\alpha 2 \mathrm{AP}$, MMP-3 and TIMP-1 were measured via western blot analysis. The histogram provides quantitative representations of $\alpha 2 \mathrm{AP}$ and MMP-3/TIMP-1 ratio (normal fibroblasts, $n=4$; SSc fibroblasts, $\mathrm{n}=7$ ). Data are presented as the mean \pm SEM. ${ }^{*} \mathrm{P}<0.01$ and ${ }^{* *} \mathrm{P}<0.05$ as indicated. $\alpha 2 \mathrm{AP}, \alpha 2$-antiplasmin; MMP-3, matrix metallopeptidase-3; TIMP-1, tissue inhibitor of metalloproteinase-1; SSc, systemic sclerosis.

A

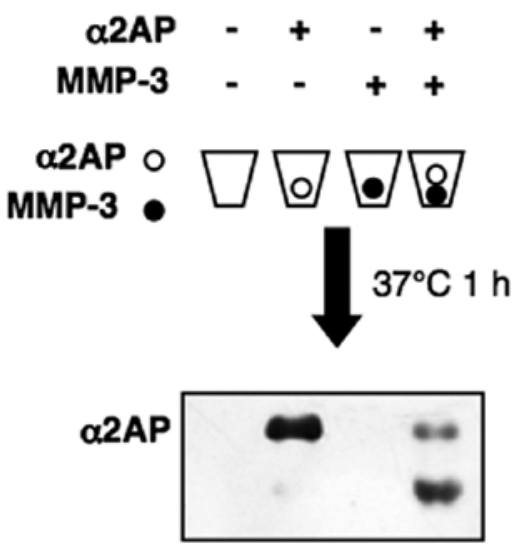

B

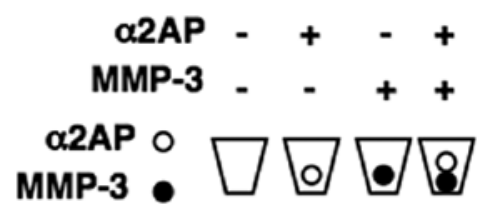

Normal FBs
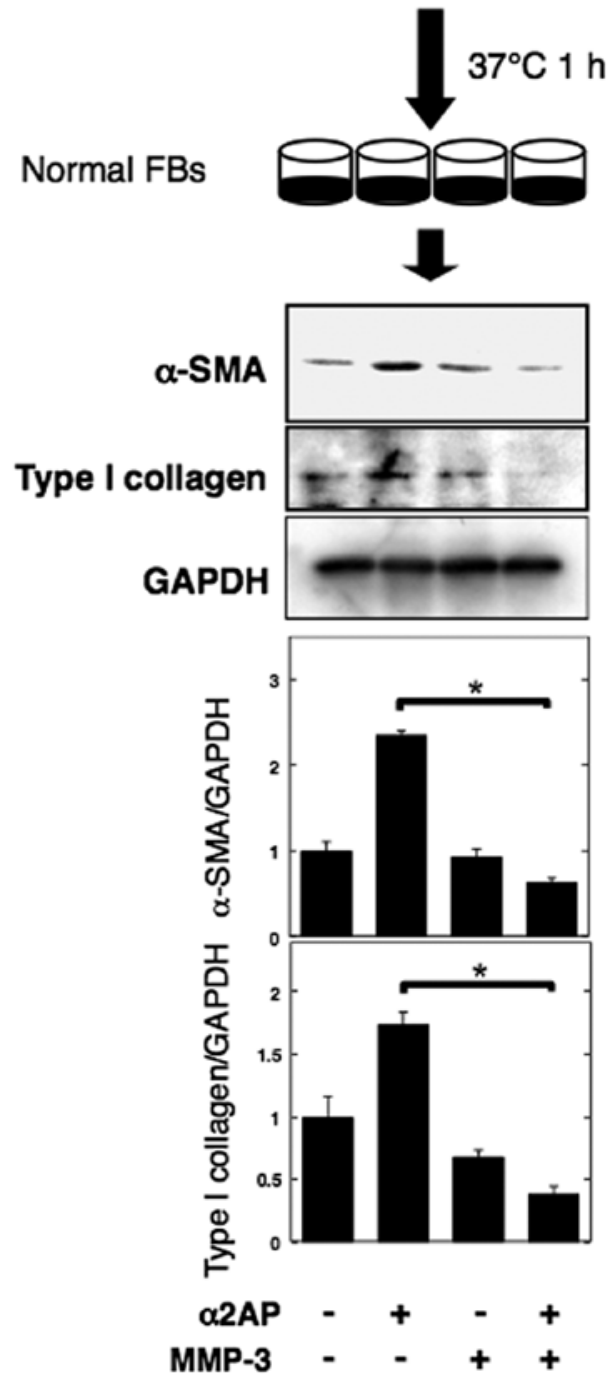

Figure 3. MMP-3-mediated $\alpha 2 \mathrm{AP}$ degradation inhibits the pro-fibrotic effects of $\alpha 2 \mathrm{AP}$. (A) Recombinant $\alpha 2 \mathrm{AP}$ was incubated with or without MMP-3 for $1 \mathrm{~h}$ at $37^{\circ} \mathrm{C}$, after which levels of $\alpha 2 \mathrm{AP}$ were examined via western blotting. (B) Recombinant $\alpha 2 \mathrm{AP}$ was incubated with or without MMP- 3 in a tube for $1 \mathrm{~h}$ at $37^{\circ} \mathrm{C}$. Normal human dermal fibroblasts were then stimulated for $24 \mathrm{~h}$. The expression of each protein was examined via western blotting. The histogram provides quantitative representations of each protein $(n=4)$. Data are presented as the mean \pm SEM. ${ }^{*} \mathrm{P}<0.01$ as indicated. MMP-3, matrix metallopeptidase- 3 ; $\alpha 2 \mathrm{AP}, \alpha 2$-antiplasmin; $\alpha \mathrm{SMA}, \alpha$ smooth muscle actin; FB, fibroblast. 
SSc FBs

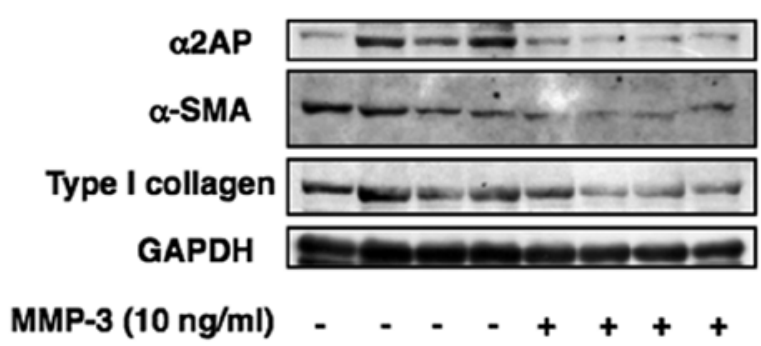

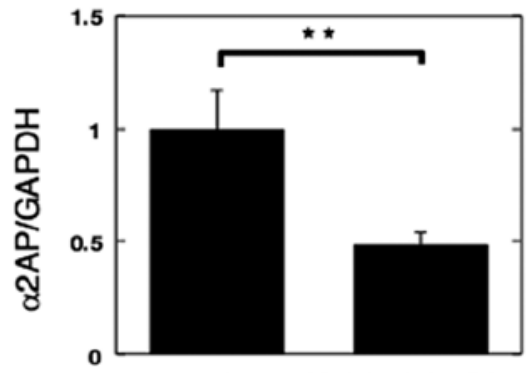
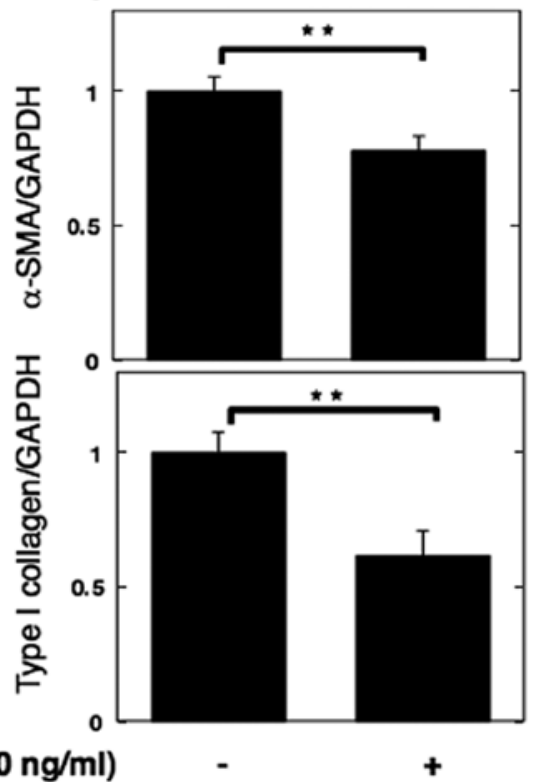

Figure 4. MMP-3 reverses the pro-fibrotic phenotype of SSc dermal fibroblasts. Human SSc dermal fibroblasts were stimulated by MMP-3 (10 ng/ml) for $24 \mathrm{~h}$. The expression of each protein was subsequently determined via western blotting. The histogram provides quantitative representations of each protein ( $\mathrm{n}=4$ ). Data are presented as the mean \pm SEM. ${ }^{* *} \mathrm{P}<0.05$ as indicated. MMP-3, matrix metallopeptidase-3; SSc, systemic sclerosis; $\alpha 2 \mathrm{AP}, \alpha 2$-antiplasmin; $\alpha \mathrm{SMA}, \alpha$ smooth muscle actin; FB, fibroblast.

\section{$\mathrm{SSc} F B s$}

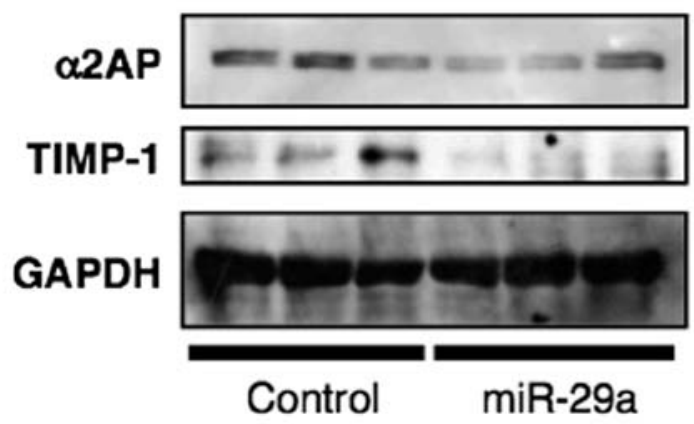

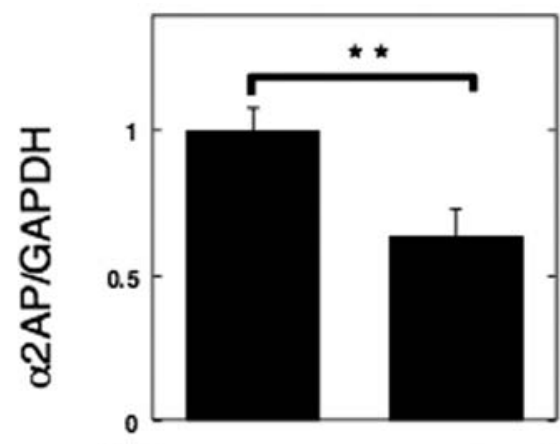

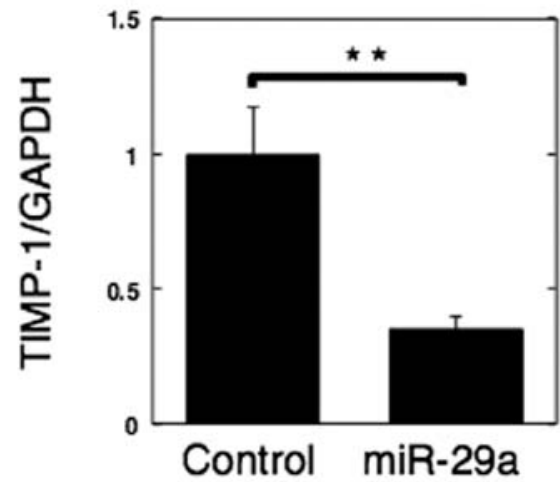

Figure 5. miRNA-29a attenuates $\alpha 2 \mathrm{AP}$ deposition in SSc dermal fibroblasts by suppressing TIMP-1 expression. Human SSc dermal fibroblasts were transfected with control or miRNA-29a and harvested $48 \mathrm{~h}$ after transfection. The expression of each protein was determined via western blotting. The histogram provides quantitative representations of each protein $(n=3)$. Data are presented as the mean $\pm \mathrm{SEM} .{ }^{* *} \mathrm{P}<0.05$ as indicated. miRNA or miR, microRNA; $\alpha 2 \mathrm{AP}$, $\alpha 2$-antiplasmin; SSc, systemic sclerosis; TIMP-1, tissue inhibitor of metalloproteinase-1; $\alpha$ SMA, $\alpha$ smooth muscle actin. 


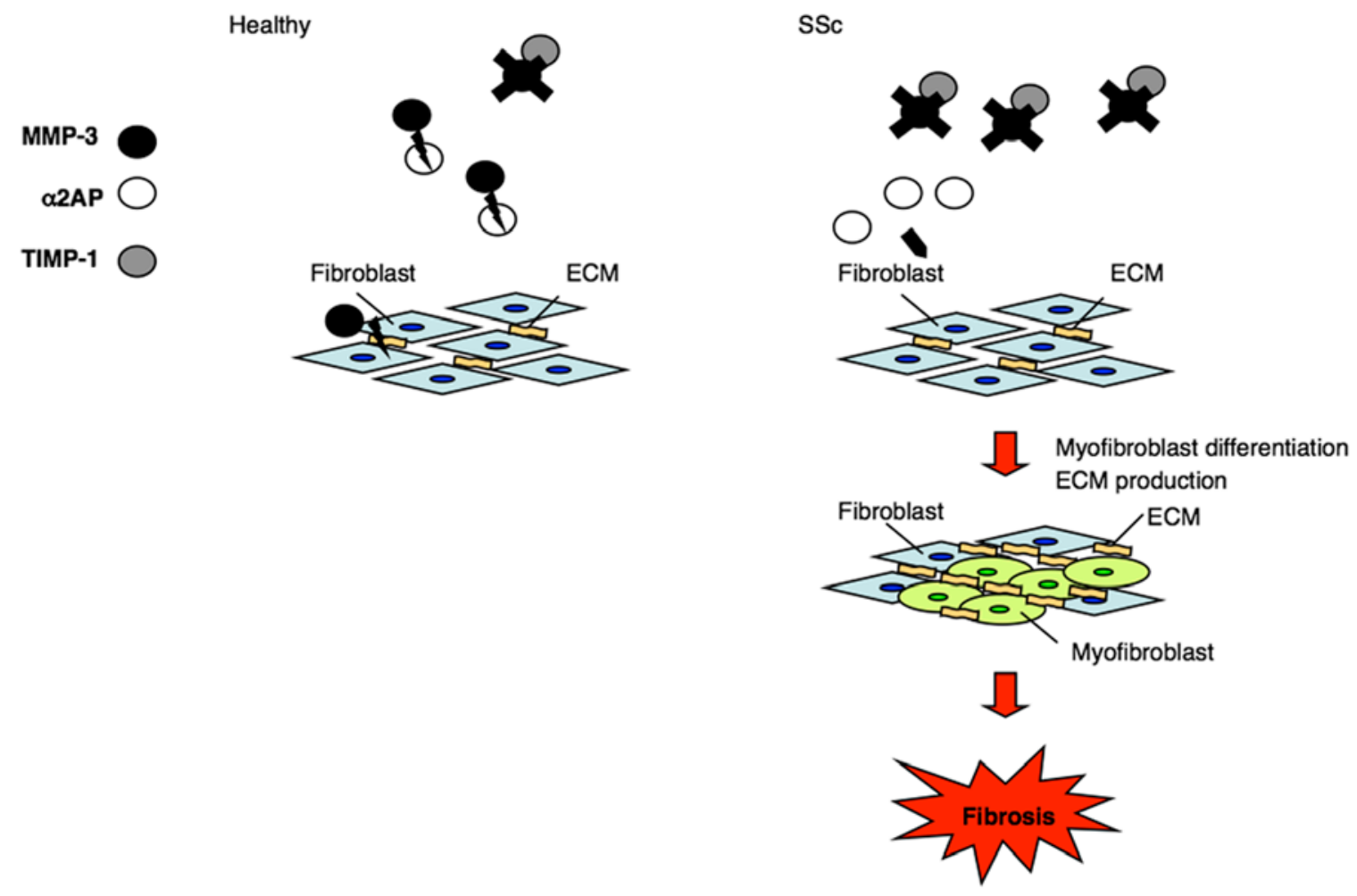

Figure 6. Role of MMP-3 and $\alpha 2 \mathrm{AP}$ in the development of SSc fibrosis. Decreased MMP-3 activity in SSc dermal fibroblasts may promote the development of fibrosis through the suppression of ECM and $\alpha 2 \mathrm{AP}$ degradation. MMP-3, matrix metallopeptidase-3; $\alpha 2 \mathrm{AP}, \alpha 2$-antiplasmin; SSc, systemic sclerosis; TIMP-1, tissue inhibitor of metalloproteinase-1; ECM, extracellular matrix.

and MMP-3 in the sera from healthy controls and SSc patients by ELISA and found no significant differences (healthy controls: $n=10$, SSc patients: $n=10)$ (Fig. 1).

a2AP expression increased while MMP-3/TIMP-1 ratio decreased in SSc dermal fibroblasts. Bonaventura et al (24) reported that the ratio of MMP-3/TIMP-1 indicates the activity of MMP-3. Hence, we next assessed $\alpha 2 \mathrm{AP}$ expression and MMP-3/TIMP-1 ratio in normal human and SSc dermal fibroblasts by western blotting (normal fibroblasts: $n=4$, SSc fibroblasts: $n=7$ ). As shown in Fig. 2, we found that $\alpha 2 \mathrm{AP}$ expression in dermal fibroblasts was increased in SSc, whereas the MMP-3/TIMP-1 ratio was reduced.

MMP-3-mediated degradation inhibits pro-fibrotic effects of $\alpha 2 A P$. Cleavage by MMP-3 inactivates $\alpha 2 \mathrm{AP}$ (23). We thus incubated $\alpha 2 \mathrm{AP}$ for $1 \mathrm{~h}$ at $37^{\circ} \mathrm{C}$ in the presence or absence of MMP-3 and showed that the enzyme degraded $\alpha 2 \mathrm{AP}$ (Fig. 3A). To assess the effect of MMP-3-mediated degradation on $\alpha 2 \mathrm{AP}$, we performed the same reactions and added the mixtures to normal human fibroblasts. Pre-incubation of a2AP with MMP-3 led to attenuation of the expression of $\alpha$-smooth muscle actin ( $\alpha$-SMA) (a marker of the myofibroblast phenotype) and type I collagen (Fig. 3B).

$M M P-3$ reverses pro-fibrotic phenotype of SSc dermal fibroblasts. Next, we stimulated SSc dermal fibroblasts with MMP-3 to investigate its anti-fibrotic effect. Consistent with the previous results, stimulation with MMP-3 decreased the expression of $\alpha 2 \mathrm{AP}, \alpha \mathrm{SMA}$, and type I collagen (Fig. 4).
MicroRNA-29a attenuates $\alpha 2 A P$ deposition in SSc dermal fibroblasts by inhibiting TIMP-1. Ciechomska et al (25) showed that miR-29a, decreased TIMP-1 expression and reversed the pro-fibrotic phenotype of SSc dermal fibroblasts. In the present study, we confirmed that miR-29a transfection in SSc fibroblasts caused a decrease in TIMP-1 expression, in line with the previous report (Fig. 5). We also showed that miR-29a caused a decrease in $\alpha 2 \mathrm{AP}$ expression in $\mathrm{SSc}$ fibroblasts (Fig. 5).

\section{Discussion}

SSc causes fibrosis of the skin and internal organs. Previously, we showed that $\alpha 2 \mathrm{AP}$ induces TGF- $\beta$ production through adipose triglyceride lipase, and is associated with myofibroblast differentiation and ECM production (9). We also showed that the expression of $\alpha 2 \mathrm{AP}$ is elevated in SSc model mice and SSc fibroblasts, and that its inactivation attenuates disease severity in SSc model mice and SSc fibroblasts $(16,17)$. These findings suggest that $\alpha 2 \mathrm{AP}$ contributes to the development of fibrosis in SSc. MMP-3, an ECM-degrading enzyme, inactivates $\alpha 2$ AP by cleaving its Pro19-Leu20 peptide bond (23). Here, we focused on $\alpha 2 \mathrm{AP}$ and MMP-3 to clarify their roles in the pathogenesis of SSc.

In this study, we showed that serum levels of $\alpha 2 \mathrm{AP}$ and MMP-3 did not vary between healthy controls and SSc patients. However, consistent with our previous findings, $\alpha 2$ AP expression in SSc dermal fibroblasts was increased. To determine whether MMP-3 contributes to the high expression of $\alpha 2 \mathrm{AP}$ in SSc fibroblasts, we measured the ratio of MMP-3/TIMP-1 as 
an indication of MMP-3 activity. Our results showed that this ratio was low in SSc dermal fibroblasts. Taken together, these data suggest that the decrease in MMP-3 activity might induce a2AP expression in SSc fibrotic tissue. Moreover, skin-specific induction and development of fibrosis may be due to increased $\alpha 2 \mathrm{AP}$ expression and decreased MMP-3 activity in tissue but not in serum.

MMP-3 inactivates $\alpha 2 \mathrm{AP}$ by proteolytic cleavage (23). Here, we confirmed that MMP-3 cleaved $\alpha 2 \mathrm{AP}$ into two fragments and attenuated the $\alpha 2 \mathrm{AP}$-induced pro-fibrotic effects, such as myofibroblast differentiation and collagen production in normal fibroblasts. In addition, treatment with MMP-3 suppressed the pro-fibrotic response of SSc fibroblasts by reducing the expression of collagen and myofibroblast markers. Moreover, MMP-3 is known to degrade ECM components, such as collagen. The decrease in MMP-3 activity in SSc dermal fibroblasts not only attenuates $\alpha 2 \mathrm{AP}$ inactivation but also promotes ECM deposition, and contributes to SSc progression (Fig. 6).

miR-29a represses TIMP-1 expression, thereby reversing the pro-fibrotic phenotype of SSc dermal fibroblasts (25). In the present study, we showed that miR-29a-transfected SSc fibroblasts exhibited a low $\alpha 2 \mathrm{AP}$ and TIMP-1 expression. Collectively, our data suggest that TIMP-1-induced MMP-3 inhibition may lead to $\alpha 2 \mathrm{AP}$ deposition in SSc.

In conclusion, we found that the activity of MMP-3 was decreased and that $\alpha 2 \mathrm{AP}$ expression was increased in $\mathrm{SSc}$ fibroblasts. Moreover, treatment with MMP-3 or suppression with a MMP-3 inhibitor, TIMP-1, reversed the pro-fibrotic phenotype of SSc dermal fibroblasts. Our results suggest that decrease in MMP-3 activity in SSc fibroblasts causes $\alpha 2 \mathrm{AP}$ and ECM deposition, and contributes to the development of fibrosis. Thus, our findings might contribute to a novel therapeutic approach for treatment of SSc.

\section{Acknowledgements}

Not applicable.

\section{Funding}

The current study was partially supported by the Takeda Science Foundation.

\section{Availability of data and materials}

The datasets used and/or analyzed during the current study are available from the corresponding author on reasonable request.

\section{Authors' contributions}

HN, YK, ES and MS designed the current study. HN, YK and ES performed the experiments and analyzed data. HN, YK, ES and MS wrote and edited the manuscript. All authors read and approved the final manuscript.

\section{Ethics approval and consent to participate}

The experiments utilizing human samples in the current study were approved by the Gifu University Graduate
School of Medicine Ethics Committee (approval no. 29-152). Written informed consent was obtained from the patients and volunteers involved.

\section{Patient consent for publication}

Not applicable.

\section{Competing interests}

The authors declare that there are no competing interests.

\section{References}

1. Gilbane AJ, Denton CP and Holmes AM: Scleroderma pathogenesis: A pivotal role for fibroblasts as effector cells. Arthritis Res Ther 15: 215, 2013

2. Collen D: Identification and some properties of a new fast-reacting plasmin inhibitor in human plasma. Eur $\mathbf{J}$ Biochem 69: 209-216, 1976.

3. Kanno Y, Ishisaki A, Kawashita E, Kuretake H, Ikeda K and Matsuo O: uPA attenuated LPS-induced inflammatory osteoclastogenesis through the plasmin/PAR-1/Ca(2+)/CaMKK/AMPK axis. Int J Biol Sci 12: 63-71, 2016.

4. Menoud PA, Sappino N, Boudal-Khoshbeen M, Vassalli JD and Sappino AP: The kidney is a major site of alpha(2)-antiplasmin production. J Clin Invest 97: 2478-2484, 1996.

5. Kanno Y: The role of fibrinolytic regulators in vascular dysfunction of systemic sclerosis. Int J Mol Sci 20: 619, 2019.

6. Kanno Y, Hirade K, Ishisaki A, Nakajima S, Suga H, Into T, Matsushita K, Okada K, Matsuo O and Matsuno H: Lack of alpha2-antiplasmin improves cutaneous wound healing via over-released vascular endothelial growth factor-induced angiogenesis in wound lesions. J Thromb Haemost 4: 1602-1610, 2006

7. Kanno Y, Kuroki A, Okada K, Tomogane K, Ueshima S, Matsuo $\mathrm{O}$ and Matsuno $\mathrm{H}$ : Alpha2-antiplasmin is involved in the production of transforming growth factor betal and fibrosis. J Thromb Haemost 5: 2266-2273, 2007.

8. Kanno Y, Kawashita E, Minamida M, Kaneiwa A, Okada K, Ueshima S, Matsuo O and Matsuno H: Alpha2-antiplasmin is associated with the progression of fibrosis. Am J Pathol 176: 238-245, 2010.

9. Kanno Y, Kawashita E, Kokado A, Okada K, Ueshima S, Matsuo O and Matsuno H: Alpha2-antiplasmin regulates the development of dermal fibrosis in mice by prostaglandin $\mathrm{F}(2 \alpha)$ synthesis through adipose triglyceride lipase/calciumindependent phospholipase A(2). Arthritis Rheum 65: 492-502, 2013.

10. Kanno Y, Kawashita E, Kokado A, Kuretake H, Ikeda K, Okada K, Seishima M, Ueshima S, Matsuo O and Matsuno H: $\alpha 2 \mathrm{AP}$ mediated myofibroblast formation and the development of renal fibrosis in unilateral ureteral obstruction. Sci Rep 4: 5967, 2014.

11. Kanno Y, Ishisaki A, Kuretake H, Maruyama C, Matsuda A and Matsuo O: $\alpha 2$-antiplasmin modulates bone formation by negatively regulating osteoblast differentiation and function. Int J Mol Med 40: 854-858, 2017.

12. Kawashita E, Kanno Y, Asayama H, Okada K, Ueshima S, Matsuo $\mathrm{O}$ and Matsuno $\mathrm{H}$ : Involvement of $\alpha 2$-antiplasmin in dendritic growth of hippocampal neurons. J Neurochem 126: 58-69, 2013.

13. Hou Y, Okada K, Okamoto C, Ueshima S and Matsuo O: Alpha2-antiplasmin is a critical regulator of angiotensin II-mediated vascular remodeling. Arterioscler. Thromb Vasc Biol 28: 1257-1262, 2008.

14. Jinnin M, Ihn H, Yamane K, Asano Y, Yazawa N and Tamaki K: Plasma plasmin-alpha2-plasmin inhibitor complex levels are increased in systemic sclerosis patients with pulmonary hypertension. Rheumatology (Oxford) 42: 240-243, 2003.

15. Kawakami M, Kawagoe M, Harigai M, Hara M, Hirose T, Hirose W, Norioka K, Suzuki K, Kitani A and Nakamura H: Elevated plasma levels of alpha 2-plasmin inhibitor-plasmin complex in patients with rheumatic diseases. Possible role of fibrinolytic mechanism in vasculitis. Arthritis Rheum 32: 1427-1433, 1989. 
16. Kanno Y, Shu E, Kanoh $\mathrm{H}$ and Seishima M: The antifibrotic effect of $\alpha 2 \mathrm{AP}$ neutralization in systemic sclerosis dermal fibroblasts and mouse models of systemic sclerosis. J Invest Dermatol 136: 762-769, 2016.

17. Kanno Y, Shu E, Kanoh H, Matsuda A and Seishima M: $\alpha 2 \mathrm{AP}$ regulates vascular alteration by inhibiting VEGF signaling in systemic sclerosis: The roles of $\alpha 2 \mathrm{AP}$ in vascular dysfunction in systemic sclerosis. Arthritis Res Ther 19: 22, 2017.

18. Chen Q, Jin M, Yang F, Zhu J, Xiao Q and Zhang L: Matrix metalloproteinases: Inflammatory regulators of cell behaviors in vascular formation and remodeling. Mediators Inflamm: Jun 12, 2013 (Epub ahead of print).

19. Van Hove I, Lemmons K, Van de Velde S, Verslegers M and Moons L: Matrix metalloproteinase-3 in the central nervous system: A look on the bright side. J Neurochem 123: 203-216, 2012

20. Nishijima C, Hayakawa I, Matsushita T, Komura K, Hasegawa M, Takehara K and Sato S: Autoantibody against matrix metalloproteinase-3 in patients with systemic sclerosis. Clin Exp Immunol 138: 357-363, 2004
21. Jinnin M, Ihn H, Asano Y, Yamane K, Yazawa N and Tamaki K: Serum matrix metalloproteinase-3 in systemic sclerosis. Arch Dermatol Res 296: 25-29, 2004.

22. Young-Min SA, Beeton C, Laughton R, Plumpton T, Bartram S, Murphy G, Black C and Cawston TE: Serum TIMP-1, TIMP-2, and MMP-1 in patients with systemic sclerosis, primary Raynaud's phenomenon, and in normal controls. Ann Rheum Dis 60: 846-851, 2001

23. Lijnen HR, Van Hoef B and Collen D: Inactivation of the serpin alpha(2)-antiplasmin by stromelysin-1. Biochim Biophys Acta 1547: 206-213, 2001.

24. Bonaventura $\mathrm{P}$, Lamboux $\mathrm{A}$, Albarède $\mathrm{F}$ and Miossec $\mathrm{P}$ : Regulatory effects of zinc on cadmium-induced cytotoxicity in chronic inflammation. PLoS One 12: e0180879, 2017.

25. Ciechomska M, O'Reilly S, Suwara M, Bogunia-Kubik K and van Laar JM: miR-29a reduces TIMP-1 production by dermal fibroblasts via targeting TGF- $\beta$ activated kinase 1 binding protein 1 , implications for systemic sclerosis. PLoS One 9: e115596, 2014. 